\title{
GAPI: Um guia online para apoio ao ensino de métodos de pesquisa com usuários
}

\author{
Izac Sidarta ${ }^{1}$, Ticianne Darin ${ }^{1}$ \\ ${ }^{1}$ Instituto UFC Virtual \\ Universidade Federal do Ceará (UFC) - Fortaleza, CE - Brazil \\ izacsidarta@gmail.com, ticianne@virtual.ufc.br
}

\begin{abstract}
Resumo. A interdisciplinaridade da Interação Humano-Computador (IHC) pode ser um desafio para o seu ensino em disciplinas introdutórias, pela necessidade de equilíbrio entre teoria e prática, a falta de maturidade dos alunos e as diferentes particularidades dos métodos de pesquisa com usuários. Dessa forma, o seguinte artigo apresenta desenvolvimento do primeiro módulo de um guia online para alunos cuja finalidade é o aprofundamento e estudo destes métodos de pesquisa em IHC.
\end{abstract}

\section{Introdução}

O ensino da Interação Humano-Computador (IHC) - uma área por natureza interdisciplinar - requer que sejam abordadas características do ser humano e das tecnologias, sob diferentes perspectivas [Churchill et al. 2016] [Rogers et al. 2013]. As habilidades e conhecimentos desenvolvidos em disciplinas de IHC vão desde técnicas clássicas de obtenção de informações das ciências sociais até o desenvolvimento de aplicações computacionais, passando por áreas como design, ergonomia, psicologia e semiótica, com o objetivo de criar soluções que atendam às necessidades e contexto humano. Dessa forma, o conhecimento de tópicos abordados pelas ciências sociais para a área de IHC é considerado essencial [Churchill et al. 2016].

Na comunidade de ensino no Brasil, os tópicos mais abordados no currículo são Introdução a IHC, Frameworks Teóricos, Avaliação, Design de Interação e Processos de Design de IHC [Guimarães and Prates 2018], com foco na integração da prática de diferentes métodos [Boscarioli et al. 2016], [Guimarães and Prates 2018]. Assim, estudos empíricos, métodos qualitativos e quantitativos de pesquisa e a análise de dados são alguns dos tópicos considerados importantes por alunos, profissionais e professores. Estes assuntos, além da sua relevância individual, afetam o estudo de tópicos chave da IHC, como Design de Interação e avaliação da interação humano-computador, incluindo usabilidade e experiência do usuário, por exemplo. [Churchill et al. 2016].Por este motivo - especialmente em disciplinas de IHC que ocorrem em cursos fortemente associados à tecnologia, engenharia e ciência da computação - é necessário prover ao aluno suporte para o desenvolvimento de habilidades que não são comuns no seu dia-a-dia por serem, tradicionalmente, habilidades próprias das ciências humanas e sociais [Leite 2010].

Este é o caso do curso cujo contexto motivou este trabalho: Bacharelado em Sistemas e Mídias Digitais da Universidade Federal do Ceará. Neste curso, alunos estudam áreas distintas do conhecimento sob uma perspectiva interdisciplinar, envolvendo tópicos da computação, comunicação e design para desenvolver competências que podem ser 
aplicadas em jogos digitais, instalações multimídia, audiovisual, design digital e sistemas interativos. Nesse contexto, o curso possui duas disciplinas de IHC, uma em caráter de obrigatoriedade, outra optativa. A primeira ( $3^{\circ}$ semestre) aborda os tópicos comuns aos currículos brasileiros [Guimarães and Prates 2018] e visa fazer o aluno refletir sobre o processo de design e compreender como os fatores humanos afetam a interação e como trabalha-los em um processo centrado no usuário. A segunda ( $7^{\circ}$ semestre) aprofunda assuntos sobre comportamento do usuário e cognição, trata abordagens de design participativo e aprofunda a prática de métodos e técnicas relacionados à usabilidade e experiência do usuário. Ambas disciplinas possuem dois créditos teóricos e dois créditos práticos.

No entanto, ao longo de oito anos ministrando as disciplinas, percebemos que no $3^{\circ}$ semestre os alunos possuem pouca maturidade para assimilar todos os aspectos relacionados aos testes com usuários. Já no $7^{\circ}$ semestre, eles não somente possuem mais maturidade para lidar com todos os aspectos da avaliação envolvendo pessoas como - em vista da perspectiva de ingressar no mercado de trabalho - estão mais desejosos de colocar em prática o que aprenderam. Porém, já esqueceram de muitos dos direcionamentos recebidos para a execução de coleta de dados e avaliações envolvendo pessoas.

Assim, o presente trabalho surge como uma forma de atender ao desafio de aprofundar o conhecimento de práticas que são apenas introduzidas nas disciplinas de IHC e apoiar os alunos na transição para a parte prática, chamando atenção aos detalhes associados a cada método. Para isso, apresentamos neste trabalho um guia online para métodos de pesquisa com o usuário, o Guia para Aprofundamento em Pesquisa com usuário em IHC (GAPI), que visa expandir o conhecimento dos alunos para literaturas além das que são comumente utilizadas nas disciplinas de IHC [Barbosa 2012]. Para este primeiro momento, foi desenvolvido o módulo sobre entrevistas e, posteriormente, serão incluídos conteúdos relacionados ao desenvolvimento de questionários e testes com usuários.

O GAPI foi desenvolvido visando as novas formas de se aprender através das redes [Porto and Santos 2019], especialmente a busca de materiais disponibilizados não só em plataformas diferentes como em formatos diferentes (artigos, vídeos, etc). Através da curadoria de conteúdos, foram sistematizadas, organizadas e agrupadas informações sobre tópicos específicos através de textos na linguagem dos estudantes, complementados por conteúdos multimídia. Nossa expectativa é que esta abordagem promova o aprendizado de forma mais acessível e prática para os alunos, e possa ser utilizada no apoio a disciplinas presenciais, bem como em práticas pedagógicas de Educação à distância (EAD).

\section{Trabalhos relacionados}

Vários trabalhos na literatura se assemelham à proposta do apresentada neste artigo, trazendo características de uma curadoria de conteúdos para a expansão dos conhecimentos e facilitação da prática de processos e métodos de IHC.

Focando em dar suporte ao ensino de avaliação de IHC, [Oliveira 2016] propõe uma ferramenta nomeada de Tool of Heuristic and Semiotic Inspection (THESI). A ferramenta tem como propósito ajudar os alunos em suas primeiras avaliações por inspeção, como avaliação heurística e inspeção semiótica. A sua função principal é uma centralização dos resultados das avaliações, servindo de apoio aos alunos para uma melhor compreensão do resultados do estudo. Dessa forma, THESI proporciona uma organização para facilitar o aprendizado da prática de inspeções de IHC pelos alunos. 
Nesta perspectiva de um produto para auxiliar e complementar o ensino de IHC, há o HCI Support Card (Cartão de suporte de IHC) [Ponciano 2019]. Trata-se de um conjunto de cartas que visa condensar informações sobre diversos tópicos, como conceitos básicos, processo de design e avaliação de IHC. O objetivo é que os alunos se envolvam mais com os conteúdos, de modo a melhorar não apenas o estudo introdutório dos tópicos abordados como também a consolidação desse conhecimento de forma duradoura.

O trabalho [Silva et al. 2019] apresenta uma curadoria de diretrizes para a avaliação e design com foco na usabilidade e acessibilidade em dispositivos móveis. Foram encontrados 369 itens em 41 publicações, entre diretrizes e heurísticas, as quais foram sistematizadas no catálogo 4All. A catalogação foi disposta de forma online, a fim de tornar mais prático e fácil o acesso ao conteúdo e auxiliar na avaliação da acessibilidade em projetos. Mesmo não sendo uma iniciativa direcionada especificamente para alunos, tendo em vista a integração com a prática dos currículos brasileiros de IHC [Guimarães and Prates 2018], trabalhos como este são de grande aplicação para o ensino.

A proposta e disponibilização online de ferramentas e conteúdos como estes são de grande valor para o processo de ensino e aprendizagem, tanto em disciplinas presenciais quanto remotas. O guia apresentado neste trabalho complementa o ensino da IHC oferecendo uma orientação guiada e aprofundada para as primeiras práticas de entrevistas.

\section{Metodologia}

Diversos autores tem organizado conteúdo confiável e abrangente sobre a aplicação de entrevistas, além de relatar e discutir os principais desafios ao utilizá-las para a coleta de dados, como [Lazar et al. 2017] e [Robson and McCartan 2016]. Para o desenvolvimento do primeiro módulo do GAPI, o conteúdo de materiais como estes foram extraídos, filtrados, catalogados e sistematizados para atender ao contexto da prática por alunos de IHC. Para isso, os passos na Figura 1 foram seguidos para construir o módulo de entrevistas.

\begin{tabular}{|c|c|c|c|c|}
\hline $\begin{array}{l}\text { Busca e filtragem dos } \\
1 \text { trabalhos }\end{array}$ & $2^{\text {Extração dos dados }}$ & 3 & $\begin{array}{l}\text { Catalogação das } \\
\text { informações }\end{array}$ & $\begin{array}{l}\text { Sistematizatização do } \\
4 \quad \text { conteúdo }\end{array}$ \\
\hline $\begin{array}{l}11 \text { trabalhos entre } \mathrm{IHC} \text { e } \\
\text { Ciências Sociais }\end{array}$ & 4 temas definidos & & 4 seções e 8 subções & 24 posts criados \\
\hline
\end{tabular}

Figura 1. Metodologia utilizada para a construção do GAPI

$\mathrm{Na}$ etapa 1 (Busca e filtragem de trabalhos), identificaram-se produções, entre livros e artigos relacionados a entrevistas como método de pesquisa, tanto na área de IHC como nas Ciências Sociais. Foram selecionados 11 trabalhos no total, 9 livros e 2 artigos, podendo serem visualizados na Tabela 1 . Iniciou-se a pesquisa através de produções indicadas por um especialista em IHC, e a partir da leitura das indicações foram selecionados novos, estes sendo também analisados pelo especialista quanto a qualidade e relevância dos materiais. Já na etapa 2 (Extração dos dados), considerou-se informações essenciais sobre entrevistas e como aplicá-las. Observando a chance de obter-se dados redundantes, conteúdos semelhantes foram comparados para avaliar sua contribuição para o tópico ou sua exclusão por, por exemplo, ser baseado em versões de trabalhos já selecionados. Facilitando não só a categorização dos dados, como a sistematização feita posteriormente.

Na etapa 3 (Catalogação das informações) foram divididas as informações em quatro seções, que serão descritas posteriormente, sendo elas: Fundamentos, Planejamento, 
Condução e Análise. Foram então extraídas as informações que abordam esses assuntos sobre entrevistas, sendo adicionadas subseções visando uma melhor organização, por exemplo, "Participantes", "Perguntas"e "Preparação dos dados". Por fim, na etapa 4, a partir das informações catalogadas foram criados e publicados de forma online e navegável 24 textos a partir do material catalogado, onde cada subseção foi contemplada com pelo menos um post. A exceção desta regra foi a seção "Fundamentos"'a qual teve seus posts associados diretamente a ela, por não possuir subseções.

\section{GAPI, o guia online}

Os textos criados para guia foram disponibilizados em formatos de posts, como em um blog (celulamultimidia.ufc.br/gapi), utilizando a plataforma Wordpress. A escolha deste formato se deu por vários fatores: a facilidade de manutenção para a inserção de novos materiais, a versatilidade no uso simultâneo e complementar de materiais em formatos diferentes (textos, imagens, áudio, vídeo) e ainda a possibilidade de tornar o guia navegável. Esta última, visando proporcionar não apenas um caminho linear para a exploração do assunto como também um acesso direto a assuntos específicos, caso o aluno queira aprender ou tirar dúvidas apenas sobre um dos conteúdos, ou parte específica deste.

\subsection{Conteúdo do guia}

Para a produção do conteúdo, utilizou-se dos dados organizados em seções e subseções A primeira contendo posts de conteúdos introdutórios sobre entrevistas, por exemplo, sobre em que casos se deve utilizá-las ou não. Três seções foram divididas, como visto na Tabela 1, dado que possuíam conteúdos que precisavam ser agrupados para uma melhor organização como também um acesso direto. As seções são: (1) Planejamento, abordando questões sobre o escopo, participantes e perguntas; (2) Condução, focada em aspectos de preparação e aplicação das sessões de entrevistas; e (3) Análise, trazendo desde a preparação dos dados, como realizar a análise até como reportar os resultados.

Tabela 1. Visão geral do conteúdo do GAPI

\begin{tabular}{|c|c|c|c|c|}
\hline \multirow[t]{2}{*}{ Tema } & \multirow[t]{6}{*}{ Seçōes | } & \multirow[t]{2}{*}{ Subseçōes } & Posts & \multirow{2}{*}{$\begin{array}{c}\text { Conteúdo } \\
\text { [Axinn and Pearce 2006]. [Kvale 2008], [Robson and McCartan 2016] }\end{array}$} \\
\hline & & & O que são entrevistas? & \\
\hline \multirow{23}{*}{ 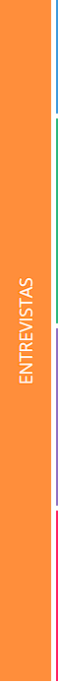 } & & \multirow{4}{*}{ FUNDAMENTOS } & Em que casos utilizar entrevistas (ou não)? & [Kvale 2008], [Lazar et al. 2017], [Robson and McCartan 2016] \\
\hline & & & Quais são os tipos de entrevista? & [Lazar et al. 2017], [Robson and McCartan 2016], [Rogers et al. 2013] \\
\hline & & & Que tipos de dados posso obter? & [Robson and McCartan 2016], [Rogers et al. 2013] \\
\hline & & & Entrevistas ou grupos de foco? & [Kvale 2008], [Lazar et al. 2017], [Robson and McCartan 2016], [Rogers et al. 2013] \\
\hline & \multirow{8}{*}{ 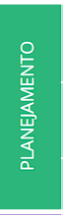 } & \multirow{3}{*}{ Escopo } & Como desenvolver o escopo e objetivo da & [Kvale 2008], [Lazar et al. 2017], [Robson and McCartan 2016], [Rogers et al. 2013] \\
\hline & & & Qual tipo de entrevista escolher? & [Lazar et al. 2017] \\
\hline & & & Como lidar com questões éticas? & [Kvale 2008] \\
\hline & & \multirow{3}{*}{ Participantes } & Quem devo entrevistar? & [Kvale 2008] \\
\hline & & & Como recrutar participantes? & [Kvale 2008] \\
\hline & & & Quantas pessoas devo entrevistar? & [Kvale 2008] \\
\hline & & \multirow{2}{*}{ Perguntas } & Como elaborar boas perguntas? & [Kvale 2008], [Lazar et al. 2017], [Robson and McCartan 2016] \\
\hline & & & Quais tipos de perguntas devo fazer? & [Kvale 2008], [Lazar et al. 2017], [Robson and McCartan 2016], [Rogers et al. 2013] \\
\hline & \multirow{5}{*}{ 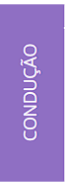 } & \multirow{5}{*}{ Entrevistando } & Como se preparar para as entrevistas? & [Kvale 2008], [Lazar et al. 2017], [Robson and McCartan 2016], [Rogers et al. 2013] \\
\hline & & & Relacionamento com o participante & $\begin{array}{l}\text { [Barbour 2008], [Kvale 2008], [Lazar et al. 2017], [Robson and McCartan 2016], [Rogers et } \\
\text { al. 2013] }\end{array}$ \\
\hline & & & Como conduzir uma entrevista? & [Lazar et al. 2017], [Robson and McCartan 2016], [Rogers et al. 2013] \\
\hline & & & Como se aprofundar nas questões? & [Kvale 2008], [Lazar et al. 2017], [Robson and McCartan 2016] \\
\hline & & & $\begin{array}{l}\text { Como registrar as respostas? } \\
\text { Como entrevistar online? }\end{array}$ & $\begin{array}{l}\text { [Kvale 2008], [Lazar et al. 2017], [Rogers et al. 2013] } \\
\text { [Lazar et al. 2017], [Robson and McCartan 2016]. [Rogers et al. 2013], [Voida et al. 2004] }\end{array}$ \\
\hline & \multirow{6}{*}{$\frac{\underline{\underline{y}}}{\frac{1}{4}}$} & Preparação dos dados & Como preparar os dados para análise? & [Kvale 2008], [Rogers et al. 2013], [Ong 2002] \\
\hline & & \multirow{4}{*}{ Execução da análise } & Como escolher o que analisar? & [Lazar et al. 2017], [Robson and McCartan 2016], [Rogers et al. 2013] \\
\hline & & & Como analisar os dados? (Parte 1) & [Robson and McCartan 2016], [Rogers et al. 2013] \\
\hline & & & Como analisar os dados? (Parte 2) & [Robson and McCartan 2016], [Rogers et al. 2013] \\
\hline & & & Posso combinar entrevistas com outros métodos? & $\begin{array}{l}\text { [Jupp 2006], [Miles and Huberman 1994], [Lazar et al. 2017], [Robson and McCartan } \\
\text { 2016], [Rogers et al. 2013] }\end{array}$ \\
\hline & & Apresentação dos dados & Como reportar os dados analisados? & [Kvale 2008], [Lazar et al. 2017], [Rogers et al. 2013] \\
\hline
\end{tabular}

Cada post foi criado seguindo uma estrutura dividida em sete partes: título, resumo do que é tratado, imagem, conteúdo, chamada para o próximo post, materiais complemen- 
tares e referências. Os três primeiros possuem o objetivo de dar as principais informações para o aluno decidir se o conteúdo é de seu interesse ou até mesmo, através das imagens descontraídas. Os títulos em formas de perguntas possuem o objetivo buscando uma melhor compreensão, além de incentivar e impulsionar o aprendizado do aluno. $\mathrm{O}$ conteúdo é onde se encontram em forma de texto as informações coletadas sobre aquele assunto. Além de serem organizadas de uma maneira lógica, buscou-se a utilização de listas, informações em destaque e subtítulos para tornar a leitura menos exaustiva.

A chamada para o próximo post se trata de um convite ao aluno para continuar ao seguinte, sendo criado um texto "apelativo"indagando o aluno sobre conhecimentos do próximo post e indicando-o. Os materiais complementares tem o objetivo de acumular diversos formatos de materiais, como podcasts e vídeos, para expandir o conhecimento. Por fim, são listadas as referências utilizadas no conteúdo para que o aluno se aprofunde pontos que forem de seu interesse, já que o GAPI não possui o objetivo de substituir a literatura, mas guiar os primeiros passos e fornecer as referências para um aprofundamento.

\subsection{Arquitetura da informação}

GAPI utilizou-se de uma sistema de organização de estrutura hierárquica, de forma larga e rasa baseada na categorização. Dessa forma, a arquitetura se deu através de apenas 3 níveis: seções, subseções e os posts. Há também a aplicação de estrutura linear, desenvolvida a partir dos posts. Essa estrutura busca facilitar a navegação e aprendizado, são utilizados sempre ao final do post um convite ao aluno para continuar para o próximo post, caso exista, através de hipertexto. Na organização, utilizou-se a organização ambígua por temas, relacionados ao processo do método. Dentro das subseções, como em seus posts, as seções foram ordenadas visando um passo-a-passo durante a pesquisa levando em conta a apresentação desses mesmos conteúdos nas publicações utilizadas para a coleta.

\section{Conclusão}

Nos últimos anos tem-se estudado quais tópicos são abordados nos currículos de disciplinas de IHC no contexto brasileiro, suas diferenças entre as diretrizes internacionais e o currículo de IHC no Brasil. Observa-se que há uma busca dos professores brasileiros por um balanço entre os assuntos abordados para realizar uma melhor integração com a prática. Assim, sendo preciso dividir o conteúdo de suas disciplinas entre fundamentos da IHC e a aplicação de processos de design, como Design Centrado no Usuário, por exemplo, onde há diversos processos e técnicas para se conhecer. O resultado disso é dificuldade de abordar os diversos assuntos relacionados a IHC em disciplinas introdutórias, principalmente aquelas que são ministradas para alunos ainda na graduação [Guimarães and Prates 2018]. Dessa forma, o GAPI busca complementar o ensino dos alunos dentro deste contexto, aprofundando o conhecimento nas práticas necessárias.

Através deste módulo de entrevistas do GAPI apresentado neste trabalho, acreditase em um aumento do aproveitamento das práticas realizadas pelos alunos. Como trabalhos futuros, é visado construção dos novos módulos, como questionários e observação de uso, e uma avaliação da experiência de uso dos alunos buscando tanto seu aprimoramento e o impacto nas atividades dos alunos. Assim, os resultados poderão gerar aprendizados para o desenvolvimento dos próximos módulos. 


\section{Referências}

Axinn, W. G. and Pearce, L. D. (2006). Mixed method data collection strategies. Cambridge University Press.

Barbosa, S. D. J. (2012). Pesquisa sobre ensino de ihc no brasil em 2012: Desafios e oportunidades. In WEIHC, pages 3-5.

Barbour, R. (2008). Doing focus groups. Sage.

Boscarioli, C., Zaina, L. A., Bim, S. A., Barbosa, S. D. J., and Silveira, M. S. (2016). Hci education in brazil from the results of the workshop on teaching of hci. In Proceedings of the 15th Brazilian Symposium on Human Factors in Computing Systems, pages 1-4.

Churchill, E. F., Bowser, A., and Preece, J. (2016). The future of hci education: a flexible, global, living curriculum. interactions, 23(2):70-73.

Guimarães, T. G. and Prates, R. O. (2018). Hci education in brazil in the light of curricula guidelines. In 2018 XLIV Latin American Computer Conference (CLEI), pages 784 793. IEEE.

Jupp, V. (2006). The Sage dictionary of social research methods. Sage.

Kitzinger, J. (1995). Qualitative research: introducing focus groups. Bmj, 311(7000):299_ 302.

Kvale, S. (2008). Doing interviews. Sage.

Lazar, J., Feng, J. H., and Hochheiser, H. (2017). Research methods in human-computer interaction. Morgan Kaufmann.

Leite, J. C. (2010). Aplicando técnicas práticas de comunicação para o design e a avaliação de ihc na formação de profissionais de computação. WEIHC/IHC.

Miles, M. B. and Huberman, A. M. (1994). Qualitative data analysis: An expanded sourcebook. sage.

Oliveira, Ana Cláudia M. T. G. e Alves, G. G. e. N. E. P. d. S. (2016). Thesi: uma ferramenta de apoio ao ensino de avaliação heurística e inspeção semiótica. WEIHC/IHC.

Ong, W. J. (2002). Orality and literacy: the technologizing of the word. 1982. London and New York: Routledge, 45.

Ponciano, L. (2019). Hci support card: Creating and using a support card for education in human-computer interaction. arXiv preprint arXiv:1909.06857.

Porto, C. and Santos, E. (2019). O livro na cibercultura.

Robson, C. and McCartan, K. (2016). Real world research. John Wiley \& Sons.

Rogers, Y., Sharp, H., and Preece, J. (2013). Design de Interação. Bookman Editora.

Silva, G. M. S., de C. Andrade, R. M., and de Gois R. Darin, T. (2019). Design and evaluation of mobile applications for people with visual impairments: a compilation of usable accessibility guidelines. In Proceedings of the 18th Brazilian Symposium on Human Factors in Computing Systems, pages 1-10.

Voida, A., Mynatt, E. D., Erickson, T., and Kellogg, W. A. (2004). Interviewing over instant messaging. In CHI'04 extended abstracts on Human factors in computing systems, pages $1344-1347$. 